Letter to the Editor

\title{
A false positive case of cardiac troponin I: Which diagnostic approach?
}

\author{
Martina Zaninotto ${ }^{\mathrm{a}}$, Aldo Clerico ${ }^{\mathrm{b}, \mathrm{c}, *}$, Ivo Casagranda ${ }^{\mathrm{d}}$, Marcello Galvani ${ }^{\mathrm{e}}$, Mario Plebani ${ }^{\mathrm{a}}$ \\ a Department of Laboratory Medicine, University-Hospital, Padova, Italy \\ ${ }^{\mathrm{b}}$ Fondazione G. Monasterio CNR-Regione Toscana, Pisa, Italy \\ c Scuola Superiore Sant'Anna, Pisa, Italy \\ d Emergency Department, Santi Antonio e Biagio e Cesare Arrigo Hospital, Alessandria, Italy \\ e Division of Cardiology, Morgagni-Pierantoni Hospital, Forli, Italy
}

\section{A R T I C L E I N F O}

\section{Article history:}

Received 7 August 2014

Accepted 9 August 2014

Available online 15 August 2014

\section{Keywords:}

Troponin

Interference

Heterophilic antibodies

Reflex testing

CK-MB

The finding of elevated cardiac troponin levels (i.e. values above the 99th percentile value of the reference population) not related to ischemic myocardial injury in patients admitted to the emergency department (ED) with suspected acute myocardial infarction (AMI), represents a relevant clinical problem, increasingly observed in the last few years, following the implementation of cardiac troponin (cTnI, cTnT) measurement with high-sensitivity immunoassays [1]. Indeed, the majority of patients (up to 70\%) admitted to the ED with more than one elevated troponin level may actually have myocardial injury unrelated to acute coronary syndrome (ACS), especially when they have an age of more than 70 years, heart failure, and/or other comorbidities [2,3]. In such patients, the incidence of "false positive" cases, i.e. the finding of troponin elevations in the absence of clinically documented myocardial injury, is actually unknown. Clinical observation however suggests that the percentage of "false positive" troponin elevations is extremely low.

In a paper recently published in the journal [4], a clinical case of a patient presenting to the ED with suspicion of AMI and non-diagnostic ECG that shows a false positive cTnI result due to the presence in the blood sample of an analytical interference caused by heterophilic antibodies, has been published. The authors suggest the adoption of an algorithm that allows the prompt detection of the supposed false-positive result. The described protocol considers the systematic measurement of $\mathrm{CK}-\mathrm{MB}$ reflex testing in all troponin-positive samples as a reliable

\footnotetext{
* Corresponding author at: Department of Laboratory Medicine, Fondazione Regione Toscana G. Monasterio, via Moruzzi 1,56126, Pisa, Italy.

E-mail address: clerico@ftgm.it (A. Clerico).
}

tool for the identification of biological and analytical interference. This reflex testing is based on the use of a well known but outdated biomarker which lacks sensitivity and specificity for the detection of myocardial injury when compared with cardiac troponin. In addition the Authors seem to ignore current international guidelines [5], which base the diagnosis of myocardial infarction, along with the documentation of objective evidence of myocardial ischemia, on the correct interpretation of serial cardiac troponin testing. In fact the analysis of the release kinetics of troponin may permit the distinction between myocardial damage caused by myocardial ischemia from myocardial injury caused by other factors and, possibly, from "false positive" troponin elevations $[5,6]$. According to these criteria, the detection of a rise and/or fall pattern of troponin with at least one value above the 99th percentile upper reference limit, is needed to make the diagnosis. It is well know, instead, that the interference of a heterophilic antibody typically causes a constant (chronic) increase in troponin values, which cannot be confused with the typical kinetic of the biomarker in AMI patients. Therefore, the interference by heterophilic antibodies should be differentiated from the huge number of clinical conditions which can cause a chronic increase in troponin levels [5]. Reflex testing is usually performed when the result of a first screening test is positive, in order to improve the specificity, although at the cost of lower sensitivity. This diagnostic strategy, therefore, should be adopted in clinical laboratory practice to improve specificity employing a second text more specific (but more expensive and time consuming) than the screening text. In the case of the proposed reflex testing [4], the cardiac specificity of the second test (i.e. CK-MB) is significantly lower than the specificity of the screening test (i.e. cardiac troponin) and significantly less sensitive for cardiac damage. As a consequence, the potential clinical advantage of using a very sensitive and cardio-specific screening test, such as the cardiac troponins, appears to be, in the proposed "reflex testing" strategy, completely lost. A huge number of clinical studies have definitively demonstrated that cardiac troponins (particularly when assayed with high-sensitivity methods) represent a more cardiac-specific and sensitive biomarker than CK-MB in cardiovascular risk stratification, both in the general population as well as in patients with cardiac disease $[1,7]$ confirming that the measurement of CK-MB is now obsolete. Moreover, considering that the incidence of heterophilic antibodies in the general population varies greatly in different studies (from 0.17 to $40 \%$ ) $[8,9]$ the reflex testing proposed, if applied systematically in all patients admitted to the ED with increased troponin values, may allow the detection of 
less than $1 \%$ of the total number of patients tested with false positive values due to the interference by heterophilic antibodies. On the contrary, this approach can entail a significant underestimation of the number of patients with cardiac damage (both ischemic and not-ischemic), thereby missing several patients at mild (or even high) cardiovascular risk of future major events, who need a strict clinical and therapeutic monitoring. The problem of analytical interference in immunoassays, well known to the laboratory professionals and widely described for several tests (TSH, D-Dimer, HCG etc. including CK-MB), is strictly method-dependent. Indeed, during the last years there has been increasing attention by manufacturers to reformulate several assays, not only those for troponin measurement, in order to reduce this type of interference [10]. Actually, the more diffuse approach deals with different technical solutions, sometime very simple and not timeconsuming (serial dilution, treatment with polyethylene-glycol, specific heterophilic blocking tube devices), that allow the rapid and safe identification of the interference, overtaking the need to modify, on the basis of a sporadic case, the recommended diagnostic biochemical algorithm that recognizes the fundamental role of troponins and the obsolescence/redundance of CK-MB assay.

\section{Conflict of interest}

The authors report no relationships that could be construed as a conflict of interest.

\section{References}

[1] Clerico A, Giannoni A, Prontera T, Giovannini S. High-sensitivity troponin: a new tool for pathophysiological investigation and clinical practice. Adv Clin Chem 2009;49:1-30.

[2] Eggers KM, Venge P, Lindahl B, Lind L. Cardiac troponin I levels measured with a high-sensitive assay increase over time and are strong predictors of mortality in an elderly population. J Am Coll Cardiol 2013;61:1906-13.

[3] Lindner G, Pfortmueller CA, Braun CT, Exadaktylos AK. Non-acute myocardial infarction-related causes of elevated high-sensitive troponin $\mathrm{T}$ in the emergency room: a cross-sectional analysis. Intern Emerg Med 2014;9:335-9.

[4] Lippi G, Ardissino D, Aloe R, Cervellin G. A false positive case of cardiac troponin I identified with CK-MB reflex testing. Int J Cardiol 2014;176:e3-4.

[5] Thygesen K, Alpert JS, Jaffe AS, et al. Third universal definition of myocardial infarction. J Am Coll Cardiol 2012;60:1581-98.

[6] Casagranda I, Cavazza M, Clerico A, et al. Proposal for the use in emergency departments of cardiac troponins measured with the latest generation methods in patients with suspected acute coronary syndrome without persistent ST-segment elevation. Clin Chem Lab Med 2013;51:1727-37.

[7] deLemos JA, deFiippi CR. Prevalence and significance of detectable troponins as measured by highly sensitive assays in the general population. Coron Artery Dis 2013;24:705-9.

[8] Vafaie M, Biener M, Mueller M, et al. Analytical false or true positive elevations of high sensitivity cardiac troponin: a systematic approach. Heart 2012;100:508-14.

[9] Lippi G, Aloe R, Meschi T, Borghi L, Cervellin G. Interference from heterophilic antibodies in troponin testing. Case report and systematic review of the literature. Clin Chim Acta 2013;426:79-84

[10] Kim WJ, Laterza OF, Hock KG, et al. Performance of a revised cardiac troponin method that minimizes interferences from heterophilic antibodies. Clin Chem 2002;48: 1028-34. 